$\begin{array}{ll}\text { Italique } & \text { Italique } \\ \text { Poésie italienne de la Renaissance }\end{array}$

XIX | 2016

Varia

\title{
Di un sonetto epistolare e alcuni sonetti filoebraici di Francesco Maria Molza
}

\section{Franco Pignatti}

\section{Q OpenEdition}

1 Journals

\section{Edizione digitale}

URL: http://journals.openedition.org/italique/430

DOI: 10.4000/italique.430

ISSN: 1663-4438

\section{Editore}

Librairie Droz

\section{Edizione cartacea}

Data di pubblicazione: 1 dicembre 2016

Paginazione: 55-86

ISBN: 978-2-600-04777-7

ISSN: 1423-3983

\section{Notizia bibliografica digitale}

Franco Pignatti, «Di un sonetto epistolare e alcuni sonetti filoebraici di Francesco Maria Molza », Italique [Online], XIX | 2016, online dal 01 décembre 2018, consultato il 07 septembre 2019. URL:

http://journals.openedition.org/italique/430; DOI : 10.4000/italique.430 
FRANCO PIGNATTI

D I U N S N E T T O E P I T O L A R E

E A L C U N S O N E T T I F I L O E B R A I C I

D I F R A C E S C M A R A M O L A 



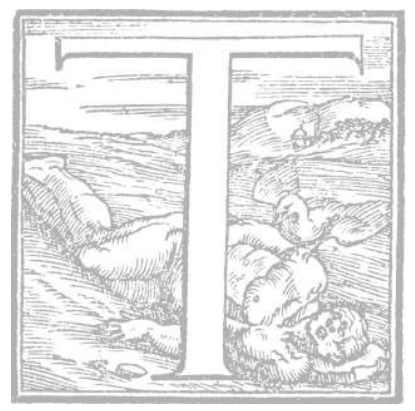

ra le numerose poesie di Molza che leggiamo nell'edizione settecentesca dell'abate Pierantonio Serassi ancora in attesa di una interpretazione soddisfacente ̀̀ il sonetto diretto a Benedetto Varchi che proponiamo di seguito insieme con la risposta, completi dell'apparato:

Mentre che lieto vi godete a l'ombra, Varchi, del vostro caro amato Lauro et con saldo Martel formate d'auro l'immagin donna che d'amor v'ingombra, 4 l'alta beltà, ch'ogni vil voglia sgombra a l'alma stanca e lei porge restauro, ricca d'un suo gentil proprio tesauro, il core in parte hor quinci hor quindi adombra 8 e duolsi pur che lunge al nostro fine fragil barchetta a duro scoglio appoggia, u' rompe il cieco suo popol perverso,

c'hor le contrade strane e peregrine

d'Egitto membra et sotto verde loggia

di Faraon trionfa in mar sommerso.

2. caro vostro $\mathrm{FR}_{2}, \mathrm{~A}, \mathrm{P}$ caro $>$ casto $\mathrm{FN}_{27}$ casto $\mathrm{FN}_{28}$ 3. et con saldo Martel formate d'auro $>$ e del suo gran valor più saldo ch'auro $\mathrm{FN}_{28} 7$. gentil suo $\mathrm{A}, \mathrm{P}$ I 2. pellegrine $\mathrm{FN}_{28}$

Sperai ben già sotto la sua dolce ombra ornar, Molza, cantando, un vivo Lauro, et con saldo Martel di forbito auro, che d'amor tutto e gioia il cor m'ingombra, $\quad 4$ opra formar di quelle che non sgombra

Morte, tal hanno contra lei restauro:

hor, non che sprima il suo doppio tesauro

lo stil mio frale, ma non pur l'adombra, et con voi duolsi ch'a non vero fine vada la bella, ch'a vil piombo appoggia speme di vetro con pensier perverso, dietro l'empio suo stuol che peregrine 
cose hor rimembra sotto verde loggia, in più reo mar che Faraon sommerso.

2. un] il $\mathrm{FL}_{3}, \mathrm{FN}_{23}$ 3. E con saldo Martel di forbito auro $>\mathrm{E}$ del suo gran valor piu saldo ch'auro $\mathrm{FN}_{28}$ 4. Che d'Amor tutto, e gioia il cor m'ingombra $>$ Che d'alta gioia il cor tutto m'ingombra $\mathrm{FN}_{27}$ Che d'alta gioia il cor tutto m'ingombra $>$ che d'alta gioia e bei pensier m'ingombra $\mathrm{FN}_{28}$ che d'alte voglie et bei pensier m'ingombra $\mathrm{FL}_{3}, \mathrm{FN}_{23}, \mathrm{FR}_{2}$ 5. quello $\mathrm{FL}_{3}, \mathrm{FN}_{23}$ 7. doppio $>$ riccho $\mathrm{FN}_{28}$ 9. noi $\mathrm{FN}_{23}$ et a $>$ ch'à $\mathrm{FN}_{27}$ I3. strade $\mathrm{FN}_{27}, \mathrm{FN}_{28}$ immembra $\mathrm{FL}_{3}, \mathrm{FN}_{23}$ ombrosa $\mathrm{FN}_{27}, \mathrm{FN}_{28}$ I 4. reo $\mathrm{FL}_{3}, \mathrm{FN}_{28}$

\section{Legenda}

A Rime del Brocardo et d'altri authori [i.e. Niccolò Dolfin e F. M. Molza], a cura di F. Amadi, Venezia, s.t., I 538 , c. $\mathrm{N}_{3 \mathrm{~V}}$

$\mathrm{FL}_{3}$ Firenze, Biblioteca Medicea Laurenziana, Conventi soppressi 430, cc. $52 \mathrm{~V}-$ 53 r (sua la grafia adottata)

$\mathrm{FN}_{23}$ Firenze, Biblioteca nazionale centrale, Panciatichiano I64, pp. 2-3

$\mathrm{FN}_{27}$ Firenze, Biblioteca nazionale centrale, II VII I43, p. 173

$\mathrm{FN}_{28}$ Firenze, Biblioteca nazionale centrale, Magl. VII 1073, p. I 89

$\mathrm{FR}_{2}$ Firenze, Biblioteca Riccardiana, Riccardiano 2803, cc. 2 I 4V-2 I $5 \mathrm{r}$

P Firenze, Biblioteca nazionale centrale, Palatino 269, c. 267v (datato I 5 aprile I6I4)

I sonetti sono trasmessi quasi sempre insieme ( $d a \mathrm{FL}_{3}, \mathrm{FN}_{23}$, $\mathrm{FN}_{27}, \mathrm{FN}_{28}, \mathrm{FR}_{2}$ ); solo quello di Molza isolato anche da $\mathrm{A} e \mathrm{P}$, per il quale ultimo, qui cosi come per le altre rime in comune, è probabile ma non dimostrabile la dipendenza da A. Per la descrizione dei testimoni rinvio alla bibliografia esistente e alla edizione delle rime di Molza che sto allestendo, di cui adotto le sigle. Per $\mathrm{FN}_{27}$ e $\mathrm{FN}_{28}$ si veda in particolare il saggio di Giuliano Tanturli sulla genesi delle due raccolte a stampa di sonetti di Varchi (De sonetti di M. Benedetto Varchi, Parte prima, Firenze, L. Torrentino, Isss e De sonetti di M. Benedetto Varchi colle risposte, e proposte di diversi, Parte seconda, ibid., I557), di cui i due manoscritti sono i codici preparatori, allestiti sotto il controllo e con interventi autografi dell' autore. ${ }^{2}$ Le due poesie che ci interessano sono stampate in Sonetti I557, p. I55, nel testo di $\mathrm{FN}_{28}{ }^{3}$.

Il testo del sonetto di Molza non pone particolari problemi, essendo le varianti di tradizione. Di rilievo è quella introdotta da Varchi al v. 2 con correzione autografa in $\mathrm{FN}_{27}$, dove subentra la qualifica della castità a proposito del Lauro, cioè Lorenzo Lenzi, a cui Varchi fu legato per l'intera esistenza da un affetto di natura omoerotica che non 
si stancò mai di presentare nelle sue rime come esperienza intellettuale e spirituale, scevra di implicazioni sensuali e origine di elevazione financo religiosa. ${ }^{4}$ In Sonetti Is ss l'aggettivo casto figura sin dal primo verso del sonetto proemiale, la cui manifesta impronta petrarchesca è stata messa abbondantemente in luce:

Quel ch'Amor mi destò casto e sincero

d'un lauro verde ne' miei più freschi anni cantai colmo di gioia, e senza inganni, se non leggiadro, almen felice e vero. ${ }^{5}$

Per il sonetto di Varchi la principale variante instaurativa di $\mathrm{FN}_{28} \grave{e}$ al $v$. 3 la rimozione del nome di Ugolino Martelli, che registra l'interruzione dei rapporti tra $i$ due letterati avvenuta non prima della fine degli anni Quaranta. Tale rimozione non ha il suo complemento nella proposta, dove il nome di Martelli non subisce damnatio, probabilmente per rispetto del testo non suo che Varchi non si senti di manomettere con un intervento pesante. In Sonetti I5s7 il ricorso a un semplice espediente tipografico permise di camuffare il nome di Martelli accanto agli altri due cui premeva dare rilievo:

MENTRE, che lieto ui godete all'ombra

VARCHI, del uostro casto, amato LAVRO,

E con saldo martel formate d'auro.

Soluzione anticipata già in $\mathrm{FN}_{28}$, che aveva agito così:

Mentre, che lieto ui godete all'ombra, VARCHI del uostro casto amato LAVRO E con saldo Martel formate d'auro. ${ }^{6}$

Nel sonetto di Varchi due errori, al $v$. s e al $v$. I3, e una variante da considerarsi erronea, al $v$. 2, congiungono $\mathrm{FL}_{3}$ e $\mathrm{FN}_{23}$; qualche problema crea la lezione di $\mathrm{FL}_{3}, \mathrm{FN}_{23}, \mathrm{FR}_{2}$ al $v$. 4, che risulta vicina a quella in cui il testo si stabilizza in $\mathrm{FN}_{28}$, mentre per il resto $i$ tre testimoni trasmettono la redazione primitiva dei due sonetti, anteriore alla revisione intervenuta in $\mathrm{FN}_{27}$ e $\mathrm{FN}_{28}$. Alv. I3 bo considerato genuina la lezione di $\mathrm{FL}_{3}, \mathrm{FN}_{23}, \mathrm{FR}_{2}$ verde loggia, contraddetta dagli idiografi, in quanto identica al sintagma del sonetto di proposta nella stessa sede, e ho lasciato a testo anche cose in quanto plausibile. 
Al futuro editore delle rime di Varchi lascio l'opzione tra mettere a testo lo stato originario o quello a cui il testo approda attraverso un processo elaborativo che coinvolge entrambi $i$ manoscritti preparatori dell'edizione a stampa. In questa sede interessa evidentemente l'occasione dialogica in cui $i$ sonetti hanno visto la luce e dunque non c'è dubbio che anche per il sonetto varchiano vada privilegiata la prima redazione, trasmessa da $\mathrm{FL}_{3}, \mathrm{FN}_{23}, \mathrm{FR}_{2}$, eccetto al v. 4, dove è stata preferita la prima lezione di $\mathrm{FN}_{27}$. Delle altre varianti di $\mathrm{FN}_{27}$ $e \mathrm{FN}_{28}$ ci siamo serviti in quanto utili a lumeggiare il significato della versione originale, piuttosto che ai fini della storia interna del testo. Cio sia detto con il corollario che la necessità avvertita da Varchi di aggiornare la testimonianza autobiografica d'antan allo stato dei fatti vigente nel momento in cui essa veniva affidata ai torchi e il pacchetto di ulteriori varianti che fu introdotto configurano, piuttosto che una diacronia, due distinte sincronie in cui si articola la storia interna del sonetto. La prima nel contesto di una corrispondenza tra due voci dialoganti, la seconda, a molti anni di distanza, registra le modifiche apportate ai testi da una sola di esse, quando l'altera pars dialogi $e$ con lei i fatti su cui si era basato lo scambio di versi si erano spenti. Ho ricostruito il rapporto tra Molza e Varchi in un'altra sede, a cui rinvio per una visione completa della vicenda, ${ }^{7}$ mi limito qui a riproporre le circostanzepertinenti allo scambio di versi su cui ci siamo concentrati, ricostruibili con una certa precisione. Dopo ilprimo incontro, a Firenze nell' agosto I536, ebbe inizio tra $i$ due una corrispondenza piuttosto fitta, dovuta all'iniziativa di Varchi, che coltivò il progetto di una edizione di rime di Molza, poi non andata in porto perle resistenze delpoeta, ma a cui Varchi si applicò con grande zelo fino al termine del I538. Alla fine di aprile I537 egli era a Roma, ospite prima degli Strozzi, nella loro casa di Borgo, poi di Lorenzo Ridolfi, anch'egli un antimediceo, ed ebbe nuova occasione di frequentare Molza. In una lettera a Pietro Vettori il 2 di giugno si definisce compare del poeta e dei primi di luglio è la notizia, ancora a Vettori, di una cena insieme. ${ }^{8}$

Varchi lasciò Roma il I2 luglio I537 con l'intenzione di raggiungere a Venezia i figli di Filippo Strozzi, Piero e Roberto, ma a Bologna lo raggiunse la notizia della disfatta di Montemurlo ( ${ }^{\circ}$ agosto) e delle esecuzioni dei prigionieri ordinate da Cosimo de' Medici, perciò prese la via di Padova, scegliendo l'esilio volontario. ${ }^{9}$ Qui al principio del I538 lo visitò Matteo Franzesi, latore della Ninfa Tiberina finalmente termi- 
nata da Molza. Il 2 f febbraio, scrivendo a quest'ultimo, Franzesi si dichiara in procinto di recarsi a Venezia per presentare il poemetto a Pietro Bembo: «il Varchi, et io andrem tosto a visitarla [scil. «sua signoria»] et a presentarle la Tiberina, la quale piace sommamente a chiunque la sente». ${ }^{\text {IO }}$

I primi quattro versi di Mentre che lieto va godete a l'ombra salutano il ricongiungimento di Varchi nella città veneta con $i$ due amici Lorenzo Lenzi (il «caro amato Lauro», v. 2) e Ugolino Martelli (il «saldo Martel», v. 3), a ricostituire una ideale unità di affetti e di studi su cui avevano avuto il loro peso le vicende politiche interne di Firenze: Lenzi era fuori Firenze già dal I532, Martelli giunse a Padova nel novembre I537. Varchi diede la notizia a Molza in una lettera databile a questo mese; in un'altra che ritengo risalga a non prima della seconda

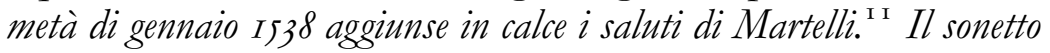
di proposta di Molza risale pertanto allo scorcio del I537 0, più probabilmente, ai primi mesi del I538 e si incastona, insieme con la risposta, nella comunicazione epistolare routinaria intercorsa tra $i$ due letterati, della quale sopravvivono radi segmenti, stante il naufragio dell'epistolario varchiano e $i$ danni irreparabili a cui sin dall'inizio apportò a quello molziano la proverbiale incuria del letterato. C'è, insomma, quanto basta per guardare a questi versi epistolari come a una testimonianza sincera del dialogo intercorso tra $i$ due letterati nei frangenti descritti e non come a un mero esercizio retorico. Nel biglietto in versi con cui raggiunge Varchi a Padova Molza saluta la nuova vita di studi intrapresa dal destinatario insieme con $i$ concittadini compagni di vecchia data, ma utilizza l'occasione per parlare anche di sé, riferendosi a una situazione che lo coinvolgeva affettivamente e intellettualmente, sulla quale Varchi era in grado di rispondere con cognizione di causa. Solo la prima strofa allude alla vita di studi intrapresa da Varchi. L'immagine che lo innamora di sé rimuovendo dal suo animo altri affetti è la filosofia o, genericamente, la sapienza. Dietro è probabilmente Rvf 94, I-2: "Quando giugne per gli occhi al cor profondo / l'imagin donna, ogni altra indi si parte», dove l' iimagin donna» è quella della donna amata, che si insignorisce del cuore dell'amante e ne scaccia ogni altro idolo. Anche «ingombra» (v. 4) va in questa direzione su autorizzazione petrarchesca, ad esempio in Rvf I0, I2: "d'amorosi penseri il cor ne ingombra»; Rvf 327, 8: "di si scuri pensieri Amor m'ingombra». "D'auro», al $v .3$ in clausola, ̀̀ ambiguo se si 
riferisca a Martel o a «l'immagin donna» al verso seguente, ma mi sembra preferibile la seconda ipotesi, non tanto perchè la saldezza non sia qualità dell'oro, quanto perché riferire il complemento di materia al simulacro, oltre a instaurare un enjambement, accentua l'immagine fabbrile di un Varchi forgiatore di preziosi manufatti di sapienza, che mi pare convenga all'elogio del destinatario che qui va in scena. Cio sebbene nella risposta Varchi modifichi la costruzione e ad essere di «forbito auro» divenga Martelli.

Con la seconda strofa Molza passa a parlare di sé e qui entro nella mia lettura del sonetto, interpretando che egli si riferisca alla donna ebrea con cui intratteneva da qualche tempo una relazione, secondo quanto si ricava da una lettera di Pietro Aretino a Bernardo Tasso del 2 ottobre I537. Cosi scrive il Divino:

Quante volte, onorato fratello, mi sono io riso e maravigliato de gli intrighi Venerei del Molza nostro? Io me ne son riso vedendogli varii, e sonmene maravigliato per i miracoli che per ciò ha fatti la vaghezza del suo sacro ingegno. Io non ho mai veduto scender la neve dal cielo senza dire: «Gli amori del tale vincan il numero di queste falde», giurando che Cupido avendo spesi per conto suo tutte le saette, era sforzato a bastonare i cori con l'arco e con la faretra. Sonmi anco stupito a pensare come il gentile de l'animo di cotanto uomo, uscendo da i santi tempi, e dei gran palazzi, abbia dato di petto ne le sinagoghe, impaniandosi d'una Ebrea, conosciuta da l'immortale per ciò. ${ }^{\mathrm{I}}$

L'«alta beltà» del $v$. 5 indica la donna mediante un sintagma di larga autorizzazione petrarchesca (ad esempio Rvf 263, I2: "L'alta beltà ch'al mondo non à pare»), cui il poeta ricorre volentieri nelle sue rime per designare le figure femminili a cui indirizza la sua devozione. La si trova, ad esempio, in Caro, che quanto scopre il nostro polo (Serassi, I, n. XCVI), dove egli indugia nell'invito ad Annibal Caroil quale rispose con Non può gir vosco, altera aquila, a volo a celebrare al suo posto la donna amata, poiché egli è impedito da «duri et lunghi affanni〉 (v. 6):

voi con l'inchiostro, onde a la morte inganni fatto più volte havete, unico et solo, cantate la divina alma beltate di lei c'ho sempre inanzi, ond'ella goda, accolta dentro a più leggiadro stile. 
Dove si avverte la presenza di $\operatorname{Rvf} 213$, 4: «e'n humil donna alta beltà divina); altrimenti in Chi mira i belli et cari occhi sereni (Serassi, III, n. XII) gli occhi della amata «son d'alta beltà ripieni» (v. 5). Ma il locus petrarchesco da convocare è Rvf 263, 9-I4, dal quale il sonetto a Varchi trae non poco lessico e la sostanza concettuale. Questi i versi interessati:

$$
\begin{aligned}
& \text { Gentilezza di sangue, et l'altre care } \\
& \text { cose tra noi, perle et robini et oro, } \\
& \text { qual vil soma egualmente dispregi. } \\
& \text { L'alta beltà ch'al mondo non à pare } \\
& \text { noia t'è, e non quanto il bel thesoro } \\
& \text { di castità par ch'ella adorni et fregi. }
\end{aligned}
$$

Troviamo qui, oltre ad «alta beltà da cui abbiamo preso le mosse, la rima oro: thesoro (auro : tesauro); «vil voglia» rigenera in Molza «vil soma» (v. s) attingendo a $\mathrm{Rvf}$ 54, I4: «fuper somma beltà vil voglia spenta», che probabilmente trascina con sé Rvf II, 4: "ch'ogni altra voglia d'entr'al cor mi sgombra» in virtu della rima; in gentil (Molza, v. 7) si avverte l'eco di gentilezza (Rvf 263, 9). Per «alma stanca» (Molza, v. 6) si dovrà guardare invece a $\operatorname{Rvf} 299$, 9-I0: «Ov'è l'ombra gentil del viso bumano / ch'òra et riposo dava a l'alma stancha» e se si vuole, con minore urgenza, Rvf 202, IO-II: «doppia colonna / porsi fra l'alma stancha e'l mortal colpos.

La presenza di Rvf 263 è ancora più scoperta in Qual donna attende in questa fragil vita (Serassi, II, n. XVII), che esula dal nostro discorso, ma che deve essere qui convocato a testimoniare la frequentazione non occasionale di questa rima sparsa petrarchesca da parte di Molza:

\footnotetext{
Qual donna attende in questa fragil vita d'honestà vivi esempi et di valore, onde lieta poi n'apra et fuor d'errore a degne lode via breve et spedita, miri il bel volto che d'amar m'invita, ivi si specchi et ivi drizzi 'l cuore, et vedrà, s'io non erro, al vero honore gentilezza di sangue insieme unita, vedrà di perle, di rubini et d'oro ugual dispregio, et sol tenersi cari
} 
que' fregi che virtù tesse et comparte:

l'alta beltate, ch'ivi ha il suo thesoro,

v'è quasi stilla d'infiniti mari,

et di sue lodi pur la minor parte.

Dunque, nel sonetto a Varchi la donna amata da Molza è dotata per nascita di virtù morali (il «suo gentil proprio thesauro», v. 7), grazie alle quali sgombra l'animo del poeta da ogni desiderio vile e lo ristora dalle avversità. Il cuore del poeta la raffigura (adombra, v. 8) dappertutto e tuttavia si duole che la fragile barca della esistenza di lei 'appoggi' a un "duro scoglio» (v. Io); contro di esso naufraga il cieco popolo di Israele, il quale, uscito dalla cattività egiziana, nella florida quiete della terra promessa rimembra la dimora nel paese straniero e consuma il suo trionfo su Faraone travolto dai flutti del mar Rosso. Interpreto fine (v. 9), nella sua ricercata indefinitezza, come oscillante tra i significati di 'termine', 'limite' e di 'destinazione', 'punto d'arrivo', anche 'destino': quindi il valore è artatamente sospeso tra il concetto di limes che la donna non può, o non è disposta, a varcare e punto d'arrivo che a lei resta precluso, e al contrario è posseduto dai due letterati dialoganti in versi. Il costrutto lunge a (v. 9), al posto di lunge da, quest'ultimo costante in Molza, trova spiegazione funzionale nella necessità di legare in sinalefe le due sillabe interessate; forse andremmo troppo oltre se vi leggessimo, meno banalmente, una sfumatura semantica che designa una distanza non per allontanamento, bensi per mancanza di arrivo a destinazione, che si adatterebbe con esattezza alla situazione in cui si trova la persona di cui si parla. Purtuttavia l'ipotesi merita di essere contemplata.

L'ermetismo dell'elocurione si appiana quando si faccia un passo ulteriore e si identifichi lo scoglio nel sacramento del battesimo, punto di inconciliabile frattura tra cristianesimo ed ebraismo. Dove si deve notare l'antitesi instaurata tra il modesto natante figura dell'esperienza esistenziale della donna, che appoggia per il momento senza patire danni agli scopula, e il naviglio dei suoi correligionari che, invece, vi naufraga. Forse ha agito qui la memoria di Rvf 264, 82: "lla mia barchetta, poi che 'nfra li scogli / è ritenuta), e, ancora, Rvf I32, IO: «in frale barca»e Rvf 235, 7: «fragil mia barcha», ma l'immagine della navigazione per l'esistenza travagliata del poeta è stilema classico e romanzo talmente consolidato da non richiedere troppi suffragi. 
L'impatto esiziale con la roccia scongiurato e la prospettiva implicita di un approdo non sono solo la soluzione ingentilita della metafora, adatta al personaggio femminile, ma vi alligna il distinguo sostanziale tra 'cecità' e 'perversità' del giudaismo, con la catastrofe che ne consegue, e l'eventualità, o l'auspicio, di una conversione in cui la donna si viene a trovare in virtù delle sue doti, e che tuttavia per ora non si compie, suscitando nel poeta il dolore di cui si è detto poco sopra (duolsi, v. 9). L'immagine di Israele che, reduce dalla cattività egiziana nella terra promessa, gode i frutti prosperi e pacifici della sua vittoria su Faraone, topicamente ritratto nel momento del rovescio anch'esso marittimo, esprime l'idea del progresso del popolo eletto lungo un cammino che poteva terminare con l'approdo alla vera religione e che invece si conclude con il naufragio di cui abbiamo detto. "Verde loggia», cosi come «verde Egitto» in S'allhor che grave servitute oppresse, v. 2 ( $d i$ cui si dirà tra poco), trae origine con ogni probabilità da memorie scritturali: Canaan è descritta topicamente nella Bibbia come terra opulenta e ospitale: «una terra dove scorre latte e miele» in Es 3, 8; Num 14, 8; Sir 46, 8, Ez 20, 6 e Is.

Il sonetto responsivo di Varchi presenta la tipica struttura a ripresa e si attiene con fedeltà alla proposta. Egli era di certo informato sulla relazione sentimentale di Molza e anzi avrà avuto modo di conoscere la donna durante il soggiorno romano, percio non ba difficoltà, partendo da questa premessa, a scendere sul piano del rapporto tra ebraismo e cristianesimo che il mittente aveva affrontato. Unica variante strutturale di rilievo è che la parte dedicata alla propria esperienza personale occupa in Varchi entrambe le quartine, mentre Molza l'aveva contenuta nella prima. Nella prima parte Varchi raccoglie la sollecitazione molziana e dichiara di avere coltivato il duplice auspicio di comporre versi per Lorenzo Lenzi godendo della sua presenza e di percorrere con il conforto e lo stimolo di Martelli la via durevole della sapienza, partorendo prodotti che non temono l'usura del tempo. Anche per Martelli tuttavia il primo getto esprime sentimenti di affettuoso entusiasmo ("che d'Amor tutto e gioia il cor m'ingombra», v. 4), destinati ad assumere nelle redazioni successive una coloritura piu castigata: amor e cor sono rimossi e introdotti bei pensieri, ma anche alta gioia, che però da $\mathrm{FN}_{28}$ sono espressioni riferite non più a Martelli, bensi a Lenzi, rimasto solo beneficiario degli elogi del poeta. 
Varchi non è da meno del poeticamente meglio attrezzato interlocutore nel frequentare il linguaggio petrarchesco e anche la sua musa riposa sull'impiego di tessere del Canzoniere, decontestualizzate e rifunzionalizzate a nuovo uso. Semmai, egli si dimostra più corrivo: la «dolce ombra» e il «vivo Lauro» arrivano, in maniera piuttosto espedita, il primo da Rvf 23, I68-69: «ché pur la sua dolce ombra / ogni men bel piacer del cor mi sgombra), probante la rima, e, con minore istanza, da Rvf I42, I: "A la dolce ombra de le belle frondi》; il secondo da $\operatorname{Rvf} 30$, 27: «l'idolo mio scolpito in vivo lauro», che inoltre, essendo Rvf 30 una sestina, ribadisce la iunctura aggettivo + lauro per altre quattro volte scolpendola nella memoria, e $\operatorname{Rvf}$ 3I8, 9: "Quel vivo lauro ove solean far nido».

Tuttavia, le aspettative di Varchi si spengono nella deminutio formulata nei vv. 7-8, dove egli proclama la sua incapacità non che di realizzare il duplice obiettivo, ma neppure di conseguirlo parzialmente. Il «suo doppio tesauro» arriva su licenza di Rvf 269, s: «Tolto m'ài, Morte, il mio doppio thesauro», dove è parola della scomparsa di Laura $e$ del cardinale Giovanni Colonna. La variante «ricco tesauro» di $\mathrm{FN}_{28}$ è dettata dalla uscita di scena di Martelli, con il conseguente riassorbirsi dei due versanti poetico-retorico e filosofico in cui Varchi aveva immaginato di versare il proprio impegno intellettuale sotto il nume del solo Lenzi. Ulteriore cellula petrarchesca è al v. 8, per cui occorre Rvf I87, 7: «nel mio stil frale».

Con il v. 9 Varchi abbandona la riflessione su di sé e passa a parlare della donna su cui Molza aveva orientato la sua attenzione nella proposta. Egli si conduole che la bella - passata dall'astratto «alta beltà) al concreto - non vada verso il fine della vera religione e poggi le sue caduche speranze su fondamenta erronee, con un impiego aggravante del termine perverso, trasferito dal popolo ebraico, cui l'aveva predicato genericamente Molza, alla sua devianza dottrinale, all'interno di un enunciato che nelle terzine volge esattamente in questa direzione.

La metafora del vetro (vv. IO-II) per la fragilità della speranz̧a è in Rvf I24, I3-I4: "Lasso, non di diamante, ma d'un vetro / veggio di man cadermi ogni speranza, / et tutti miei pensier' romper nel mezzo", ma si arricchisce qui del paragone con il piombo, di cui prevale la qualifica di viltà rispetto ai metalli nobili (l'oro del v. 3) e dunque la metafora veicola il concetto che sia impossibile per l'ebraismo aprirsi a un cammino verso la vera religione, anche in presenza di personali doti 
d'eccezione come quelle che la donna in questione possiede. La terzina finale introduce il giudizio di empietà (v. I2), più grave di quello di cecità, e dipinge il popolo ebraico nella terra promessa immerso in un metaforico mare addirittura più rio di quello in cui era stato sommerso Faraone, dunque incapace di distaccarsi dall'errore in cui versa, laddove la proposta aveva tenuto aperta la prospettiva di evoluzione e conciliazione dell'ebraismo con il cristianesimo.

Le due varianti presenti nel sonetto di Varchi al $v$. I3 in $\mathrm{FN}_{27}$ e $\mathrm{FN}_{28}$ accentuano, a mio avviso, lo snaturamento dell'immagine biblica che Molza aveva presente e del senso che vi aveva sotteso. La sostiturione di cose con strade, forse mutuata da contrade, in Molza al v. I2, ripristina il senso della rimembranza delle trascorse vicissitudini nel tempo felice (la cattività egiziana o anche l'erranza nel Sinai), che con la caduta di Egitto era andato un po' perso. É pero piuttosto la scelta di ombrosa al posto di verde, forse con l'intenzione di stabilire una polarità con ombra al v. I, che trasferisce l'idea di opulenza e di dono di Dio insita nell'aggettivo originario, verde, a quella oziosa e supervacanea di un locus amoenus in cui trascorrere le ore rammemorando le prove superate, senza accorgersi che l'illusione del raggiungimento della terra promessa cela invece il fallimento di una falsa religione che non porta alla salvezza. In conclusione, Varchi non si produce in una consolatoria per la querela di Molza addolorato dalla indisponibilità della donna amata a convertirsi passando per le acque lustrali del battesimo, piuttosto dà corso alla dichiarazione che tale prospettiva è illusoria e senza esito.

Un altro sonetto di Varchi a Molza, questa volta di proposta, risalente alla medesima congiuntura che abbiamo illustrato, insiste sul medesimo punto in maniera più ottimista. Il testo trasmesso da $\mathrm{FN}_{27}, p .88$, e $\mathrm{FN}_{28}, p .89$, è vergine da interventi, per cui le modifiche che sono presenti in Sonetti I55), p. I06, furono introdotte in un interpositus. Do il testo di $\mathrm{FN}_{27}, \mathrm{FN}_{28}$ e in apparato le varianti di Sonetti I55s:

Molza, che pien di quelle usanze antiche vergate ogn'hor di bei pensier le carte, onde si fan conserve in ogni parte dell'honorate vostre alte fatiche, quanto m'incresce Amor per me vel diche non poter, poi che 'l ciel da noi vi parte, 
venir là vosco ove 'l popol di Marte

hebbe tanto le stelle un tempo amiche;

gite, dunque, felice, e non vi gravi

in mio nome portar salute humile

al mio buon Caro, et al gran Casa vostro;

sì dagl'error di sua gente empi e gravi

tragga 'l chiaro di voi cortese inchiostro

la bella donna al nostro santo ovile.

5-6. poi che le stelle a me sempre nemiche / mi vietano, hor che 'l ciel da noi vi parte 8 . hebbe l'armi e le muse un tempo amiche 9. dunque] prego

Credo che il motore delle due modifiche più importanti risieda nella necessità di rimuovere Amor al v. 5, avvertito come sconveniente: lo spostamento di stelle dal v. 8 al v. 5 comportò il risarcimento al v. 8 con la coppia «l'armi e le muse», adatta al soggetto di cui è parola. La locuzione «popolo di Marte» ha la sua probabile origine in Rvf 53, 26: «che se 'l popol di Marte I dovesse al proprio honore alzar mai gli occhi» e Tr. Fame, II, 2 ("presa a mirar il buon popol di Marte»), gode di discreta auge nella lirica cinquecentesca e fu sfruttata anche da Molza come perifrasi per indicare gli abitanti di Roma richiamandosi alle loro nobili origini. ${ }^{13}$ Varchi sottopone insomma il dettato primitivo alla moralizzazione, in questo caso un po' proude, che informa tutta la rilettura della produzione precedente al momento di avviarla ai torchi. Al v. g la sostituzione del nesso conclusivo dunque con l'interiezione prego, calco del quaeso latino, va soltanto nella direzione di una maggiore eleganza del dettato.

Il nome di Annibal Caro ricorre nelle lettere di Varchi di questo periodo e salutiper Giovanni Della Casa e Paolo Giovio sono nella chiusa di una lettera notturna che Varchi scrisse a Molza nel novembre I537:

Io non vi voglio più infastidire, e anche per dir il vero ho un gran sonno, però farò fine, detto che vi arò e pregato che mi mandiate a ogni modo le stanze [la Ninfa Tiberina] e mi raccomandiate a messer Giovanni della Casa e al Como gentile e a tutti gli amici e padroni. State sano. ${ }^{\text {I4 }}$

Poiché non ho trovato il sonetto responsivo di quello appena riportato tra quelli di Molza e Varchi non lo incluse in Sonetti I557, inclino a credere che esso non sia stato inviato al destinatario, forse per resipiscenza del suo autore dinanzi alla qualità scadente di questi versi, o 
forse perché battuto sul tempo dal sonetto di proposta molziano al quale Varchi rispose, non potendosi sottrarre alla corrispondenza in versi con il più titolato interlocutore, al quale chiedeva allora le sue poesie per la pubblicazione. Quest'altro sonetto varchiano si esaurisce in una interpretazione piuttosto schematica della formula epistolare, toccando quattro punti ordinatamente distribuiti nelle strofe: allocuzione elogiativa iniziale, separazione per volere del fato avverso, saluti per $i$ due sodali romani di Molza, infine l'auspicio che la donna ebrea amata dal destinatario abbracci la religione cristiana. Ritengo che questo sonetto preceda gli altri due e sia la traccia di un esperimento abortito di avviare da parte di Varchi uno scambio, fallito in primo luogo nell'obiettivo centrale in questo tipo di testi, consistente nello sperimentare un linguaggio allusivo, che esclude gli estranei dal dialogo tra $i$ due protagonisti, come avviene nella successiva proposta di Molza. Se si accetta questa ricostruzione, si deve tuttavia constatare che fu Varchi a toccare per primo il tema della relazione sentimentale di Molza con la donna ebrea e della sua conversione, il che vuol dire che tale fatto doveva essere l'esperienza saliente nella vita di Molza in quel momento e Varchi registrò la cosa nei suoi versi.

L'episodio biografico su cui sia Varchi sia Molza convergono autonomamente nell' avviare la corrispondenza in versi permette di recuperare, oltre l'occasione epistolare, un manipolo di sonetti del cui rapporto con la testimonianza della lettera di Pietro Aretino citata sopra si era accorto addirittura Adolfo Gaspary, ma dopo di lui, a quanto mi risulta, la notizia è rimasto lettera morta. Dando prova di notevole acribia, Gaspary nella sua Storia della letteratura italiana scriveva:

I molti amoretti del Molza erano quasi proverbiali; Pietro Aretino diceva (Lett. I, I67), che Cupido doveva ormai colpire i cuori coll'arco e colla faretra, avendo consumato contro di lui tutti i suoi dardi. Nonché di altre si era allora ( 1537 ) innamorato di una ebrea e questo nuovo soggetto arricchì la sua poesia di certi motivi, che non provengono dal repertorio generale. Così egli supplica Dio di donar la sua grazia a codesto bel rampollo della schiatta maledetta, di sollevarlo al cielo coll'acqua santa (son.: Poiché la vite, onde Israel fioria); le fa il complimento che, se il suo popolo non si fosse lasciato sfuggire il regno, ella ne sarebbe ora la regina (son.: Da la radice che fiorir devea), e l'amore desta in lui interessamento per tutto Israele, per cui spera misericordia e lume da Dio (son.: Alto fattor del mondo e Dentro a ben nato). ${ }^{15}$ 
Con maggiore precisione, alla bella ebrea amata da Molza va ricondotta una sequenza di quattro sonetti trascritti di seguito nell'autografo $\mathrm{C}$ (Roma, Biblioteca Casanatense 2667, posteriore al 26 luglio I54I e molto probabilmente anteriore all'agosto I543), nn. I7-20, e nel fratello T (Milano, Biblioteca Ambrosiana, Trotti 43I), nn. 55-58, tra i quali troviamo due dei quattro segnalatida Gaspary. ${ }^{16}$ Ne riporto il testo di seguito conservando la numerazione di $\mathrm{C}$ con cui li identificheremo d'ora in avanti:

17

Da la radice che fiorir devea

e 'l ciel empiendo di soave odore saldar le piaghe del commun errore, allhor che 'l mondo più di ciò tacea, uscendo la gran verga, fu a Giudea lo scettro tolto e 'l pregio suo maggiore, i seggi disturbati et svèlto il fiore, onde già il grido d'ogni altezza havea.

Se ciò non era, hoggi il Giordani ${ }^{17}$ a voi con l'onde serve partirebbe il piano, et le cime alzarebbe irte il Carmelo.

Degna mai certo ella non hebbe poi alma di ciò, fin ch'ogni dolce et piano lume non versò in voi cortese il cielo.

Esci di tua magion et lieta oblia, alma gentile, ogni paterno affetto, et fia del re del ciel sommo diletto la rara tua beltà, la leggiadria.

Verranno al tuo valor per lunga via le genti accese dal soave aspetto, et conto in breve ad ogni alto intelletto andrà di te il valor, la cortesia.

Mira le prime due ben nate piante, cui sopra d'Israel crebbe le sponde l'alto Fattor, et diè lor pregio eterno, et di gir ti fie lieve ognihor più avante, cogliendo il fior di cui arida fronde tieni hor et quella stringe horrido verno. 
S'allhor che grave servitute oppresse il popol vostro là nel verde Egitto, ove più volte fu sì forte afflitto, ch'al gran dolor quasi per forza cesse, vi produceva il ciel, assai men spesse eran le piaghe d'amendui et dritto camino, oltra il fatal corso prescritto, havean di gir al fin l'alte impromesse. Ch'una rivolta de' begli occhi santi, d'honestà, di letitia alteri nidi, snodato havrebbe a Faraone il core et ciò ch'ei non credette a i segni tanti del saggio duca et a' suoi spessi gridi, sarebbe hor vostra preda et vostro honore.

Poi che la vite ond'Israel fioria gravi sdegni del ciel giusti sfrondaro, di suoi pregi maggior sì la spogliaro, che tanto giacque quanto già salia; hor stella, non so come, amica et pia di germe l'orna sì soave et chiaro, che 'l passato destino, empio et amaro, onde a forza piegò, crescendo oblia. Signor, tu che di lei già fostù seme, et ombre ne trahesti indegne et felle, come al gran Padre et a te stesso piacque, il vago ramo, onde convien ch'io treme, da le squalide sue sterpi ne svelle, e al ciel l'inalza con le tue sante acque.

La tradizione di questi sonetti è asimmetrica; riporto $i$ dati in forma abbreviata, rinviando alla legenda in nota. I nn. I7-I8 sono testimoniati oltre che dall'autografo $\mathrm{C}$ e da T, soltanto da $\mathrm{P}$ e da Serassi, descritti. I nn. 19-20, invece, hanno avuto diffusione ampia, in larga parte condivisa. Essi sono trasmessi insieme anche da $\mathrm{BI}, \mathrm{CV}_{\mathrm{I} 4}, \mathrm{FN}_{13}, \mathrm{SI}_{5}{ }^{{ }^{1}}{ }^{8}$ $\mathrm{SI}_{6}{ }^{1}{ }^{19} \mathrm{WR}, \mathrm{RD}_{3}$. Inoltre, il $n .20$ anche da $\mathrm{F}_{\mathrm{I}}, \mathrm{RDR}_{\mathrm{I}}$ e dalle sillogi 
di rime spirituali $\mathrm{RSpB}, \mathrm{RSpSa}$, tutti descritti, direttamente o indirettamente, da $\mathrm{RD}_{3}$. Il n. 20 è anche in $\mathrm{SI}_{1}$, anch'esso descritto. $D a \mathrm{RD}_{3}$ (per C 19) e da $\mathrm{RDR}_{\mathrm{I}}$ (per C 20) dipende l'edizione settecentesca Rime di Francesco Maria Molza, a cura di Antonio Bellucci Gentili e Giovanni Ballirani, Bologna, C. Pisarri, I7I3, da cui discende a sua volta Serassi. Quest'ultimo recupera gli inediti nn. I7-I8 da $\mathrm{P}$, ma non ricostituisce il quartetto perché la scelta editoriale di Serassi è di tenere separati editi e inediti. ${ }^{20}$ Dunque, riepilogando, C $17-I 8$ restarono consegnati alle carte del poeta e trovarono registrazione solo in $\mathrm{C}$ e nell'omologo $\mathrm{T}$, che fu esemplato posteriormente a $\mathrm{C}$ sugli stessi antigrafi da cui era stato tratto l'autografo; C 19-20 furono pubblicati, e non è detto che ciò sia avvenuto per volontà dell'autore. Nella diversa vicenda di questi ultimi spicca ulteriormente l'ospitalità negata al n. I9 $d a \mathrm{~F}_{\mathrm{I}}$ e $\mathrm{RDR}_{\mathrm{I}}$, le due grandi sillogi di rime compilate sulle edizioni a stampa precedenti, che furono i piu autorevoli istituti attraverso $i$ quali la lirica italiana del XVI secolo fu veicolata alle epoche successive e alle altre letterature europee. Poiché $\mathrm{F}_{\mathrm{I}}$ coincide (salvo poche eccezioni) per Molza con il canone stabilito da $\mathrm{RDR}_{\mathrm{I}}$, l'ostracismo inflitto al n. Ig ha il suo responsabile in Lodovico Dolce, editore di $\mathrm{RDR}_{\mathrm{I}}$, a cui forse dispiacque riproporre $i$ due sonetti di contenuto affine recensiti in $\mathrm{RD}_{3}$. Al di là di queste vicissitudini, sulle quali può essere considerato ozioso soffermarsi avendo a disposizione l'autografo, i sonetti citati sono importanti perché sono il documento della occasione biografica su cui lo scambio epistolare in versi da cui siamo partiti si innesta. Al centro di questi versi è la donna di religione israelitica amata da Molza, alla quale il poeta si rivolge come eletta rappresentante del suo popolo in virtù delle sue doti spirituali tanto quanto dei suoi pregi estetici, esortandola alla conversione.

Il n. I7 è occupato per due strofe da una ampia perifrasi che indica in Cristo il messia che avrebbe dovuto concludere la storia del popolo eletto e poiché gli ebrei non lo riconobbero posero le premesse della loro servitù e della loro umiliazione. ${ }^{2 \mathrm{I}}$ "Gran verga» (v. 5), cioè virgulto, è Cristo, in quanto nato dalla stirpe ebraica, che avrebbe dovuto redimere dal peccato originale (il «commun errore», v. 3) il proprio popolo e i gentili in un destino di comune salvezza. Tutto ciò fa da prodromo all'elogio contenuto nella seconda terzina, formulato in maniera generica, ma in sostanza consistente in una comparazione sorprendente tra l'avvento di Cristo come frutto misconosciuto dell'ebraismo e la donna amata da 
Molza, in cui il cielo ha riversato dopo tanti secoli $i$ suoi doni. "Lume» (v. I4) è l'influsso astrale, come in $\mathrm{Rvf} 7$, 5-6: "uet è si spento ogni benigno lume / del ciel» (e anche $\operatorname{Rvf}$ I42, Io e $\operatorname{Rvf} 325,72)$, ma «dolce et piano lume» (vv. I3-I4), presente solo qui in Molza, ricorda $\mathrm{Rvf} 276$, I3-I4: "(i)l dolce et amoroso et piano / lume degli occhi miei», cioè Laura, $e$ in $\operatorname{Rvf} 72,2$; Rvf 106, 8; Rvf 142, 3I «dolce lume» indica lo sguardo di Laura, Laura stessa in Rvf I63, 9. Dolce e piano segnalano doti di benevolenza e facilità in Rvf 42, I: "Ma poi che 'l dolce riso bumile et piano». Per cui in tale elezione divina si riversa la materia sentimentale conveniente alla destinataria dell'elogio poetico e predominante sulla missione spirituale, il cui senso e contenuto resta qui, cosi come nel resto della suite, arcano.

Il n. I contiene l'invito alla conversione, che sarà occasione per la donna di dare pieno compimento e pubblicità alle sue virtù e di divenire iperbolicamente modello per le genti. La beltà e la leggiadria (v. 4), con il «soave aspetto» (v. $\sigma)$, insistono sull'aspetto fisico e sui modi, mentre valor e cortesia ( $v .8)$ alludono a pregi interiori e di non espressa connotazione muliebre. E probabile agisca in sottofondo la memoria di Rvf 26I, Qual donna attende a gloriosa fama, che, prossima al valico centrale strutturante il libro petrarchesco di rime, contiene l'elogio delle virtù superiori di Laura e le cui parole della rima $\mathrm{B}$ coincidono per tre quarti (cortesia: leggiadria: via; manca mia, in enjambement: mia / nemica) con quelle della rima A di Molza. La conversione è dichiarata espressamente nella seconda terzina, attraverso la metafora vegetale dominante per l'intero quartetto di poesie, con la fioritura del ramo sterile dell'ebraismo, di cui la donna rappresenta il rampollo vitale. Per mezzo della stessa metafora nella prima terzina due personalità (s. Pietro e s. Paolo?) sono menzionate per il loro valore esemplare di come l'ebraismo veterotestamentario debba essere letto nella prospettiva di una continuazione nel cristianesimo.

Nel n. In Molza si spinge a immaginare che lo sguardo della donna avrebbe ammansito Faraone, con il risultato di risparmiare ad egiziani $e$ ad ebrei le sofferenze delle piaghe mandate da Dio sull'Egitto e di facilitare ai secondi il ritorno nella terra promessa, modificando in meglio la storia del popolo eletto. In questo modo, grazie al suo fascino coniugato a qualità morali, la donna si trova equiparata al ruolo svolto da Mosè, con una singolare disinvoltura nel rileggere le Sacre Scritture dal punto di vista dei propri affetti o, per meglio dire, di utilizzarle 
spregiudicatamente per formulare una galanteria iperbolica, spoglia di risvolti dottrinali.

Una invenzione dello stesso conio si legge in C 130 (trasmesso inoltre solo da $\mathrm{T}$ e dai descritti $\mathrm{P}$ e Serassi), in cui con audacia il poeta si spinge a immaginare che il pianto della donna (questa volta una cristiana), in grado di molcere gli animi anche più duri, avrebbe piegato financo il decreto divino e risparmiato a Cristo il supplizio della croce, di fatto cancellando l'evento da cui dipende la redenzione dell'nomo. La scaturigine di quella che appare di nuovo una galanteria sesquipedale rivolta alla donna corteggiata sono le lacrime da lei versate (v. 5$)$, per le quali il poeta confeziona questo concetto leggiadro e sorprendente. Il suo carattere blasfemo non sfuggi all'abate Serassi, che pubblico il sonetto detrattone il v. I0. ${ }^{22}$ Ecco il testo completo:

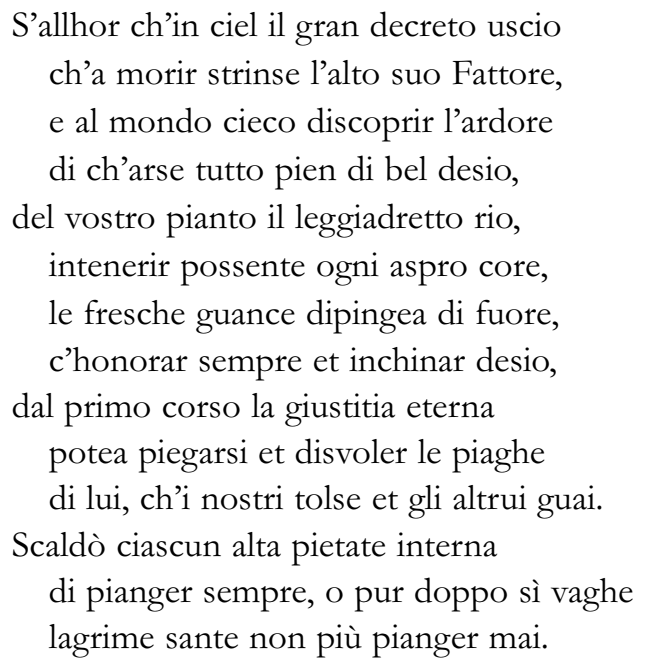

Mi soffermo sul v. II dove mi pare che l'espressione «i nostri tolse e gli altrui guai» sia equivalente a «saldar le piaghe del commun errore» di C 17,3 , cioè sottintenda una visione ecumenica della salvazione che riguardi oltre $i$ cristiani anche $i$ giudei, che a tale salvazione si sottrassero per loro insipienza, mentre ne sono implicitamente esclusi i musulmani che, non provenendo dal ceppo comune del giudaismo, sono infedeli. Cosi, anche per tramite di questa cellula, il sonetto si ricollega al gruppetto filoebraico di C I7-20 e ciò potrebbe essere considerato anche come indizio di prossimità nella composižione. 
C 20 chiude il quartetto dei sonetti riproponendo $i$ temi già illustrati della sventura che ha colpito Israele in conseguenza del rifiuto del Vangelo, e dell'influenza celeste che ha dotato la donna amata dal poeta di virtù tali da riscattare la cattiva sorte del suo popolo. L'allegoria della vigna come simbolo del popolo eletto è di casa nella tradizione sia vetero sia neotestamentaria. Fonte di Molza potrebbe essere Isaia 5, I-7, dove si narra di una vigna coltivata con la massima cura dal suo proprietario, che invece di uva produce agresto. Perciò egli decide di ridurla in rovina e lasciare che vi crescano rovi e pruni: «Ebbene, la vigna del Signore degli eserciti è la casa d'Israele; gli abitanti di Giuda la piantagione prediletta. Ne attese rettitudine, ed ecco invece nequizia, giustizia, ed ecco invece grida di angoscia». Di tale vigna sfrondata per punizione divina Cristo fu il nuovo seme e per volontà sua e del Padre l'ha mondata degli aspetti indegni e ingiusti con il suo martirio. A Cristo si rivolge la preghiera del poeta affinché tragga dalla vigna rimasta sterile di Israele (per «squalide sue sterpi», v. I3, cfr. Rvf 3I8, 5: «mostrando al sol la sua squalida sterpe») il «vago ramo» di cui egli è innamorato, cioè illumini con la grazia la donna ed ella entri nella religione cristiana attraverso il sacramento del battesimo. Cosi il breve ciclo si conclude con l'esplicita menzione dell'atto decisivo cui il poeta chiama la donna, nei termini non del rinnegamento della religione dei padri, ma dell'adesione alla discendenza illuminata del giudaismo quale ai suoi occhi appare il cristianesimo.

La presenza dei quattro sonetti uniti in $\mathrm{C}$ e $\mathrm{T}$ toglie ogni dubbio sul fatto che essi debbano stare insieme. Tuttavia, altre poesie della medesima origine si trovano altrove in $\mathrm{C}$ e $\mathrm{T}$; ciò significa che Molza non coltivò l'intenzione di costituire una raccoltina delle poesie composte per la stessa destinataria e si limitò a mantenere unite, o a riunire al momento della trascrizione in $\mathrm{C}$, le quattro che veniamo da leggere. La seconda eventualità potrebbe spiegare la tradizione differente che si ̀̀ illustrata sopra, cui $i$ sonetti andarono incontro a coppie. Un altro sonetto collocato altrove sia in C (n. IOI) sia in T $(n .46)$, dedicato nientemeno che alla Natività, menziona ancora la donna di C I7-20 ("alma gentil», v. I3; cfr. Rvf I46, 2: "alma gentil cui tante carte vergo») e presenta la medesima metaforica arborea che aveva fornito l'ossatura a C I7-20: 
Dentro a ben nato aventuroso chiostro di bella donna et di virtute ardente, cassa di luce anchor la nostra gente litigò col diletto popol vostro, e se 'l divino et honorato inchiostro, che promessa non falla, in ciò non mente, tempo verrà che, le discordie spente, torni lieto Israel ad esser nostro.

Così talhor un tronco solo ordisce di sé doppia colonna a gli altrui fianchi, et dui rami maggior sospinge fuore:

il vostro è secco et sol di voi fiorisce alma gentil, et o pur non le manchi dal ciel benigno et pretioso humore.

C IOI è addirittura più radicale di C I7-20 nel proporre un parallelo tra ebraismo e cristianesimo, coinvolgendo questa volta la nascita di Gesù. Il testo vive di alcuni sintagmi pesanti del Canzoniere: la Vergine è "bella donna», con un sintagma ad alta frequenza per designare Laura; «virtute ardente» è in $\operatorname{Rvf} 283$, 3: «spirto più acceso di vertuti ardenti»; Rvf I46, I: «O d'ardente vertute ornata et calda»; Rvf 337, 6: «ogni bellezza, ogni vertute ardente», ma Molza se ne appropria e vi ricorre con una certa frequenza (Serassi, I, n. IX, 7; n. XXXVIII, 2; n. XXXIX, s; II, n. LXXX, 2; n. CIX, 3). Affiora al v. Io, decontestualizzata, la memoria di $\operatorname{Rvf}$ I26, 6: «a lei di fare al bel fiancho colonna».

Ma l'interesse del sonetto non riposa su questi referti stilistici, bensi sulla notevole dell'utero di Maria in cui la matrice ebraica e l'embrione da cui verrà la rivelazione del Vangelo, coesistono in un rapporto che viene descritto come conflittuale in potenza, poiché la rivelazione non ha ancora avuto luogo e «la nostra gente» è appunto «cassa di luce» (v. 3 , cfr. Rvf 294, 6: "Amor de la sua luce ignudo et casso»). E tuttavia è ribadita l'elezione del popolo ebraico al quale la nascita di Gesù è stata annunciata dalla profezia veterotestamentaria. L'annuncio che il ceppo secco dell'ebraismo dopo tanti secoli di sterilità è divenuto di nuovo vitale dando fuori la donna amata da Molza stabilisce un parallelo cristologico tra lei e Gesù al di fuori di ogni proporzione accettabile.

A fianco a $\mathrm{C}$ IoI bisogna mettere $\mathrm{C} 44(=\mathrm{T} 50)$, ad esso legato per l'insistenza sul medesimo tema, ma anche per importanti affinità formali che avvicinano strettamente le due poesie: 


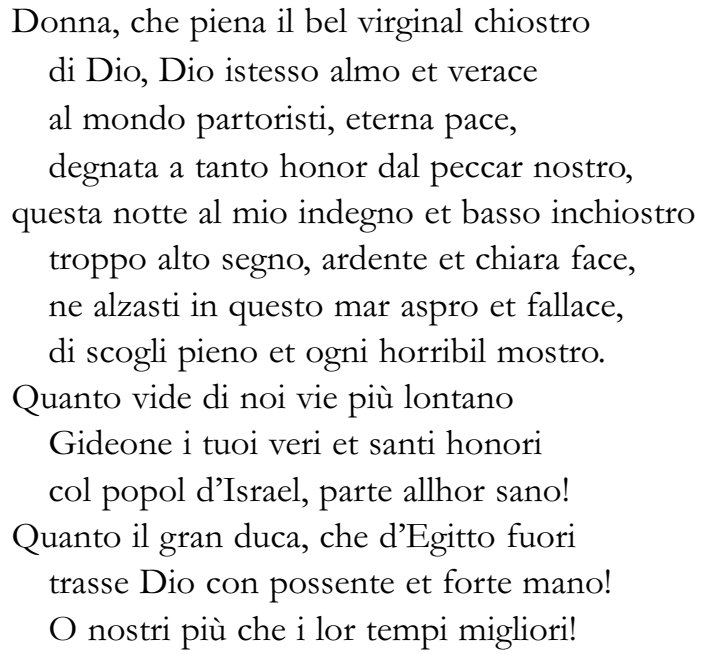

Non solo i due incipit sono molto simili, ma la rima A è la stessa di $\mathrm{C}$ Ior e le parole rima di $\mathrm{C} 44$ sono le medesime di Già donna, or dea, nel cui virginal chiostro di Bembo (qui mostro al v. 8 è però voce del verbo 'mostrare'), in cui la gestazione del Redentore è esplorata come primo stadio del processo che condurrà alla sconfitta di Satana e alla salvezza dell'umanità dal peccato:

Già donna, or dea, nel cui virginal chiostro, scendendo in terra a sentir caldo e gelo, s'armò, per liberarne, il re del cielo, da l'empie man de l'aversario nostro.

La scaturigine di questi versi è in quelli della canzone alla Vergine petrarchesca: «ricorditi che fece il peccar nostro, / prender Dio per scamparne, / bumana carne al tuo virginal chiostro» ( $\operatorname{Rvf} 366,76-78)$, e un'eco di $\operatorname{Rvf} 366$ si avverte, indipendentemente dal filtro bembiano, nella prima quartina del sonetto di Molza: ai vv. 2-3 "Raccomandami al tuo Figliuol, verace / homo et verace Dio, / ch'accolga 'l mïo spirto ultimo in pace» ( $\operatorname{Rvf} 366,135-37)$. Al v. 4 si riconosce, inoltre, la memoria di $\operatorname{Rvf}$ I3 $_{3} 8:$ : «che fosti a tanto honor degnata allora».

Ma Molza ricava poco dal sonetto bembiano, che dopo la prima strofa si snoda in maniera piuttosto rituale nella invocazione alla Vergine come figura adiutrice, cui il poeta si rivolge alle soglie della vecchiaia formulando propositi di nuova vita (vv. I3-I4: "romai de la mia vita / si volge il terzo e cinquantesim'anno»). Al centro dei versi di Molza è 
l'incarnazione: il poeta dichiara la sua inadeguatezza a celebrare la notte in cui Maria ha dato alla luce Gesù e ai vv. 6-8 il Salvatore è guida e scorta dell'umanità, metaforicamente faro che indica il tragitto sicuro nel mare tempestoso e incerto della nostra esistenza, con riproposta della immagine marittima presente nel sonetto epistolare a Varchi. Non sfugge il tono suasorio di questi versi, che si spiega con il fatto che la destinataria è di religione israelitica. Alla tematica cristologica subentra infatti nelle terzine l'evocazione di due personaggi veterotestamentari che ripropongono il contenuto profetico dell'Antico Testamento e la convinzione che Gesù sia il messia annunciato nelle Scritture.

La presenza di Gedeone è piuttosto singolare. Nel racconto biblico di Giudici 6-8 egli distrugge l'altare di Baal eretto dal padre, sostituendolo con uno al Signore, poi con uno stratagemma sconfigge i Madianiti, che opprimono Israele. Egli attacca il loro accampamento di notte con uno scelto manipolo di guerrieri, che seminano il panico nelle file nemiche suonando $i$ corni e agitando fiaccole. I Madianiti, sorpresi nel sonno, si mettono a gridare e a correre, colpendosi alla cieca tra loro. I vv. IO-II alludono alla eliminazione del culto idolatrico compiuta da Gedeone e al fatto che per ordine del Signore egli selezioni progressivamente le sue numerosissime truppe, fino a ridurle ad appena trecento uomini. Lo scopo teologico di tale drastica riduzione è mostrare che l'impresa è una guerra santa guidata e vinta solamente da Dio e non dalla potenza militare umana.

La liberazione dal dominio dei Madianiti fa di Gedeone una figura paragonabile a Mosè, che aveva affrancato gli ebrei dalla cattività egiziana. L'accostamento è suggerito in Giudici 6, I3: "Dove sono tutti quei prodigi del Signore, di cui ci parlano i nostri padri, quando dicono che Egli ci fece uscire dall'Egitto?»); il suo agire è costellata da dubbi e dalla richiesta al Signore di dargli piu segni della sua elezione, in maniera non dissimile da quanto avviene nell'Esodo con le esitazioni che attanagliano Mosè e le piaghe che persuadono Faraone a lasciare partire gli ebrei.

L'impiego di Mosè correlato alla Natività, sebbene piuttosto audace, non costituisce un problema eccessivo, se si considera che nella esegesi cristiana egli è interpretato anagogicamente come figura Christi, poiché è allegoria della liberazione dell' anima dal peccato. Non mi risulta che una lettura analoga sia stata proposta per Gedeone. In Purgatorio 25, 
I24-26 il racconto di Giudici 7, 25 è modestamente utilizzato come exemplum del peccato di gola: «e delli Ebrei ch'al ber si mostrar molli, / per che no i volle Gedeon compagni, / quando ver Madian discese $i$ colli) ( $i$ soldati che, condotti da Gedeone alla fonte di Carod, per bere si inginocchiarono e portarono l'acqua alla bocca con la palma della mano furono esclusi, coloro che invece lambirono l'acqua con la lingua senza inginocchiarsi, dimostrando di essere sempre pronti al combattimento, furono prescelti).

Una volta accettato il collegamento tra Mosè e Cristo e dunque con la Natività, l'ulteriore anello costituito da Gedeone a me pare si spieghi meglio nella prospettiva della storia di Israele che di una esegesi cristiana, perciò sono propenso a credere che agisca qui l'influenza della bella e dotta ebrea di cui Molza si era invaghito e abbia avuto luogo, in minoribus, un accostamento (non un sincretismo) tra l'esegesi allegorica della Bibbia propria della tradizione cristiana e la lettura storicosimbolica a cui si attenevano gli ebrei. L'enunciato lapidario con cui la poesia si chiude suggella il tutto con il ribadire il primato della rivelazione sull'annuncio veterotestamentario.

Dal punto di vista della tradizione, con C 44 e IOI ci troviamo dunque dinanzi a uno stato di cose analogo a quello che abbiamo esaminato per C I7-20. I due sonetti, affini per contenuto e linguaggio, tanto da potere essere considerati gemelli, furono trascritti separati sia in $\mathrm{C}$ sia in $\mathrm{T}$ ed ebbero una storia differente. Mentre $\mathrm{C}$ IOI restò consegnato a $\mathrm{C} e \mathrm{~T}, \mathrm{C} 44$ trovò ospitalità in $\mathrm{FOS} e$ in $\mathrm{RD}_{3}$; da quest'ultimo si originò una folta discendenza, soprattutto a stampa, che gli diede ampia visibilità: $\mathrm{P}, \mathrm{SI}_{\mathrm{I}}, \mathrm{RDR}_{\mathrm{I}}, \mathrm{F}_{\mathrm{I}}, \mathrm{RSpB}, \mathrm{RSpS}, \mathrm{RSpSa}$, Rime I7I3, Serassi. ${ }^{23}$ Dove una breve chiosa merita l'accoglienza del sonetto, cosi come abbiamo visto sopra per C 20, nelle sillogi di Rime spirituali sfornate dall'industria tipografica non solo veneziana a partire dalla metà del secolo per rispondere al nuovo orizzonte culturale controriformistico: considerata la genesi di queste poesie è quasi umoristico che esse siano approdate a un tale esito.

Un terzo sonetto sulla Natività, C 62, ha una tradizione più folta: BI, $\mathrm{C}, \mathrm{FN}_{\mathrm{I}}, \mathrm{FOS}, \mathrm{MV}, \mathrm{RN}_{\mathrm{I}}, \mathrm{T}, \mathrm{RD}_{3}$ e $i$ descritti $\mathrm{P}, \mathrm{SI}_{\mathrm{I}}, \mathrm{F}_{\mathrm{I}}, \mathrm{RDR}_{\mathrm{I}}$, $\mathrm{RSpB}, \mathrm{RSpSa}$, Rime I7I3, Serassi. ${ }^{24}$ Anch'esso presenta un contenuto piuttosto singolare:

Cedi pur, giorno, et men volgendo altero de la gran face, che 'l tuo lume appanni 
la notte soffri e 'ncominciarsi gli anni

da lei comporta, rimirando al vero:

sotto lei nacque chi Giovanni et Piero

col ciel le reti cangiar fece e i panni,

e, 'ncisi i nervi a Belzebub e i vanni,

le porte aperse del vetato impero.

Stupì Natura et al gran parto intenta,

tosto ch'a se medesma fé ritorno,

sciolse la lingua quasi in tai parole:

«Godi Giudea, senza fin contenta,

poi ch'eletta da Dio sei per soggiorno

de l'immortal sua vera unica prole».

Molra accosta il motivo della luminosità della notte in cui Gesù venne al mondo presente in numerose poesie celebranti la Natività tra XV e $X V I$ secolo, ${ }^{25}$ che però, dato il contesto, potrebbe avere raccolto la suggestione di Salmi 139, I2: "Ebbene, non sono oscure per te le tenebre, e la notte risplende come il giorno, come le tenebre così è la luces. Il tema è proposto attraverso il concetto piuttosto lambiccato che il giorno (la «gran face», al v. 2, è il sole), dovrà cedere il passo alla notte, perché vinto in lucenterza, e accettare che il computo degli anni avvenga partendo dalla notte del dì della Natività e non dal giorno. Cristo è indicato come colui che ba convertito gli apostoli ed è risorto ( $v v$. 7-8). La Natura stessa contempla trasecolata la nascita di Cristo, al punto da dover tornare in sé prima di poter formulare la frase con cui il sonetto si chiude in maniera piuttosto clamorosa. Giudea ( $v$. I2) credo vada interpretata alla lettera come definizione geografica e non, per slittamento metonimico, il popolo che vi abita, ma resta il contenuto che la regione in cui ha avuto corso la storia di Israele è anche il teatro dell'avvento del messia annunciato dalle Scritture, cosa di cui Giudea è invitata a gioire.

Sul motivo del valore profetico dell'Antico Testamento è incentrato C 97. Anche in questo caso siamo di fronte a una tradizione bifida, del tipo di quelle che abbiamo descritto finora: oltre che da $\mathrm{T}$, e dai descritti $\mathrm{P}$, Serassi, il sonetto è trasmesso dai due codici tra loro molto vicini e autorevoli $\mathrm{BI} e \mathrm{FN}_{13}$, i quali restituiscono oltretutto la medesima didascalia: "Del Molza per il populo hebreo» («popolo Hebreo» $\left.\mathrm{FN}_{\mathrm{I} 3}\right):^{26}$ 


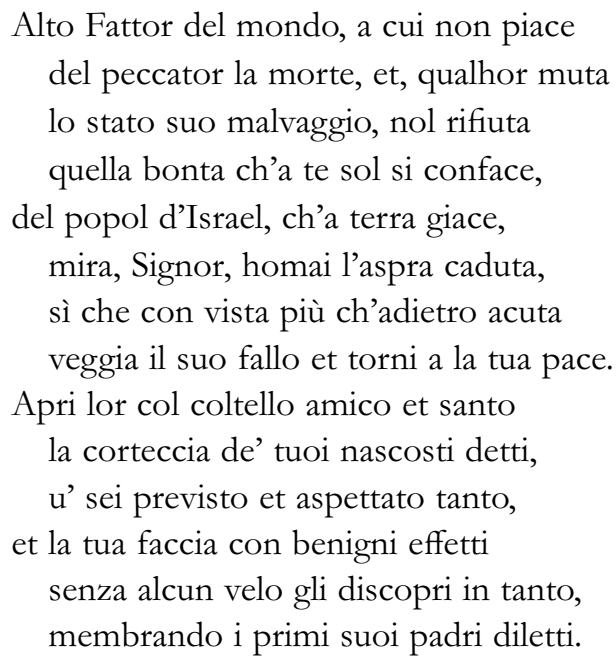

Ritorna l'anelito al recupero dell'ebraismo al cristianesimo, all'interno di una visione irenica per cui la benevolenza di Dio si estende verso la religione da cui il cristianesimo è nato e il messaggio di salvezza contenuto dell'Antico Testamento si deve rivelare nella parola dei patriarchi e dei profeti, $i$ «padri diletti» (v. I4), venerati anche dai cristiani.

Con ciò si chinde il dossier sulle rime filoebraiche di Molza, con l'auspicio che una nuova tessera biografica o un rinvenimento testuale permettano di gettare ulteriore luce su questi versi e di risolvere i passaggi rimasti poco chiari. Il loro bilancio sul piano teologico è assai magro, specie se si confrontano con alcuni sonetti scambiati con Vittoria Colonna e con altri incentrati sul sacrificio della croce o su temi spirituali, nei quali allignano contenuti più sodi, proposizioni vicine alle tesi evangeliche, memorie scritturali che rivelano una frequentazione non superficiale della Bibbia.

Più consistente il valore letterario di questi versi, in cui Molza si serve con maestria della koiné petrarchesca per trattare contenuti inediti e personalissimi, con un atteggiamento non tanto minaccioso per la ortodossia, quanto assolutamente peculiare per la leggerezza e l'irriverenza con cui tratta le Sacre Scritture. Che Molza abbia accolto questi testi in $\mathrm{C}$, che è una silloge della sua produzione grosso modo dell'ultimo decennio, trascritta quando la sua vita volgeva al termine, vuol dire che non gli sembrarono imbarazzanti sul piano del contenuto e neppure 
della vicenda biografica a cui erano legati. Che in $\mathrm{C}$ siano rimasti legati solo $i$ quattro sonetti che avevano come destinataria la donna ebrea amata è dunque un omaggio a posteriori a quella relazione; la dispersione in $\mathrm{C}$ degli altri sonetti che abbiamo recensito non sorprende se si guarda al criterio con cui fu costruita la silloge, che reca altri episodi del genere. Neppure sorprende in ultima istanza che il sonetto a Varchi non sia stato incluso, sebbene $\mathrm{C}$ accolga diverse poesie di corrispondenza (senza le corresponsive: si è nominato sopra $\mathrm{C} 35$, Caro, che quanto scopre il nostro polo), in quanto la composizione della silloge $\grave{e}$ aliena da un disegno unitario e dal proposito di fornire un ritratto esauriente della produzione del poeta.

In chiusura, resta da presentare un ulteriore lacerto di poesia epistolare varchiana che coinvolge Molia, questa volta in maniera problematica. In Sonetti I555, p. 85, si legge la seguente poesia:

Hor dura pioggia a mezzo aprile, hor folta nebbia che asconde e bagna, la dolente alma mia trista accompagna da sì lieti pensieri a pianger volta, né ragion cura più, né vede, involta nel duol, quanto qui piace opra di ragna essere, e come in van prega e si lagna di lei che tutti ancide e null'ascolta.

Così da voi lontan gran tempo omai, arbor del sol, tra nebbie e pioggie e venti meno la vita in dolorosi guai,

e, se 'l ciel meco a pruova e gl'elementi piangon ne i più bei mesi e giorni gai, chi porrà fine o quando a i miei lamenti?

"Arbor del sol», al v. Io, è Lorenzo Lenzi, a cui appartiene questo epiteto che non è che una perifrasi per il lauro, in quanto pianta sacra ad Apollo. Il sonetto conclusivo di Sonetti Isss ne fa uso nella prima strofa, stabilendo l'arco cronologico entro cui deve essere collocata la vicenda sentimentale che costituisce il nerbo della raccolta:

Da voi felice e senza alcun affanno hebbe principio il mio cantare, et hora felice e lieto in voi fornisce ancora, arbor del sole, al ventottesimo anno. 
Il sonetto a $p .85$ risulta nel complesso pervio e non solleva ostacoli seri alla comprensione. Dei codici preparatori, $\mathrm{FN}_{28}$, p. 65, presenta il testo della stampa, invece in $\mathrm{FN}_{27}, p$. 89, dove il sonetto viene di seguito a Molza, che pien di quelle usanze antiche $(p .88)$, il v. Io reca a testo la lezione: "Molza gentil tra sterpi, e sassi, e venti», cassata e sostituita nel margine dalla lezione di $\mathrm{FN}_{28}$ e della stampa, vergata da Varchi nella sua tipica corsiva qui particolarmente corrente. La variante a testo in $\mathrm{FN}_{27}$ è palesemente fuori posto e Varchi non fece qui che correggere uno svarione del copista, ma la lettura del sonetto fu attenta perché il $v .7$ reca una microcorrezione: o si lagna $>$ e si lagna, recepita anch'essa da $\mathrm{FN}_{28}$. Tutto chiaro, dunque; resta da capire la scaturigine del frustulo molziano venuto accidentalmente a rimpiazzare l'«amato Lauro» di Varchi e con ciò svelare una probabile pagina ignota del dialogo in versi tra $i$ due letterati.

Franco Pignatti 
I. Delle poesie volgari e latine di Francesco Maria Molza corrette, illustrate, ed accresciute colla vita dell'autore scritta da Pierantonio Serassi, Bergamo, P. Lancellotti, I747-1754, I, n. XLIII. L'unico che si è cimentato con l'«enigmatico scambio di sonetti» è stato Domenico Chiodo, che ha fornito una interpretazione in chiave di allegoria politica, dalla quale mi discosto (Varchi rimatore: modi e forme della poesia di corrispondenza, in Benedetto Varchi I503-I565. Atti del convegno (Firenze, I6-I7 dicembre 2003), a cura di V. Bramanti, Roma, Edizioni di Storia e Letteratura, 2007, pp. 93-1 87, pp. 168-70). Per completezza cito il mio F. Pignatti, Benedetto Varchi e il progetto di edizione delle rime di Francesco Maria Molza, in Varchi e altro Rinascimento. Studi offerti a Vanni Bramanti, a cura di S. Lo Re, F. Tomasi, Manziana, Vecchiarelli, 20I3, pp. 8I-Iо9, pp. 94-97, dove mi sono limitato a porre il problema senza avanzare una interpretazione.

2. G. Tanturli, Una gestazione e un parto gemellare: la prima e la seconda parte dei "Sonetti" di Benedetto Varchi, in «Italique», VII (2004), pp. 43-100.

3. Da Sonetti I 557 dipende Opere di Benedetto Varchi, II, Trieste, Lloyd Austriaco, I 858 , p. 957 .

4. Tanturli, Una gestazione e un parto gemellare, cit., p. 47.

5. De sonetti di M. Benedetto Varchi, Parte prima, Firenze, L. Torrentino, I 555 , p. 3; cfr. B. Huss, "Cantai colmo di gioia, e senza inganni». Benedetto Varchis Sonetti (prima parte) im Kontext des italienischen Cinquecento-Petrarkismus, in «Romanistisches Jahrbuch», LII (200I), pp. I33-57, pp. I40-43; Tanturli, Una gestazione e un parto gemellare, cit., pp. 49-50; L. Paolino, Il 'geminato ardore' di Benedetto Varchi. Storia e costruzione di un canzoniere 'ellittico', in «Nuova Rivista di Letteratura Italiana», VII (2004), pp. 233-314, pp. 249-52.

6. Ho dato i testi in trascrizione diplomatica perché nel secondo verso è presente una figura per l'occhio che ritengo imputabile all'autore: VARchi/LAVRo, alla quale $\mathrm{FN}_{28}$ e Sonetti I 557 danno risalto, rispetto alla scrizione anodina di $\mathrm{FN}_{27}$ e di tutti gli altri testimoni: Varchi/Lauro.

7. Pignatti, Benedetto Varchi e il progetto di edizione delle rime, cit.

8. Benedetto Varchi, Lettere, a cura di V. Bramanti, Roma, Edizioni di Storia e Letteratura, 2008 , pp. 53 e 58.

9. Il io agosto Mattio Franzesi, appena tornato da avere trionfato con Molza, Claudio Tolomei e un certo Antognotto, gli scriveva una lettera malinconica, alla quale Benedetto Busini aggiunse un post scriptum di suo pugno (Lettere a Benedetto Varchi (I530-I563), a cura di V. Bramanti, Manziana, Vecchiarelli, 20I 2, p. Ioo). Antognotto deve essere l'Antoniotto Da Bene nominato, di nuovo accanto a Tolomei, tra gli amici di Molza nella lettera di Varchi da Venezia del gennaio i 537 (VArchi, Lettere, p. 62, per la datazione cfr. infra nota i I4) e, ancora solo con il nome, in una di Annibal Caro sempre a Molza del 28 giugno i 543 (AnNibal Caro, Lettere familiari, a cura di A. Greco, Firenze, Le Monnier, I957-196I, I, p. 276). Sui movimenti di Varchi in questo frangente cfr. S. Lo Re, Politica e cultura nella Firenze cosimiana. Studi su Benedetto Varchi, Manziana, Vecchiarelli, 2008, pp. 467-69. 
ı. Firenze, Biblioteca nazionale, II VII I 29, pp. 54-55; cfr. Pignatti, Benedetto Varchi e il progetto di edizione delle rime, cit., p. Ioo.

i i. VARCHI, Lettere, p. 62. Ritocco la datazione al novembre-dicembre i 537 data da Bramanti in VARCHI, Lettere, p. 64 sulla base del fatto che Varchi tranquillizza il destinatario circa le voci giunte a Roma di ottave scritte da Aretino contro di lui («messer Pietro Aretino non ha fatto stanze né altro mai contra vostra signoria, anzi fa professione d'amare le vertù vostre e di conoscerle») e una lettera di Annibal Caro a Varchi del io gennaio I 538 ritrae un Molza preoccupato proprio per questa notizia: «Il Motta [lo scrittore apostolico Bernardino Motta] mi disse iermattina ch'egli l'avea trovato ch'andava tutto affannato, per avere inteso che v'erano stanze dell'Aretino stampate contra lui, e cercava d'esse: di che mi meraviglio, sendo vero, ché mi parea che 'l Molza avesse l'Aretino per amico» e di nuovo più avanti: «Parlai col padre Molza, e delle stanze contra lui; non credo sia altro: pure, avvertitemi» (CARO, Lettere familiari, n. [27-28 bis], parr. 5 e I 3 ). La lettera di Varchi a Molza con le rassicurazioni è dunque posteriore a questa data e contestualmente Varchi scrisse anche a Caro: «A compare Annibale ho scritto e egli vi conferirà alcuna cosa, se bisognerà» (VARCHI, Lettere, p. 65). La lettera cui Caro risponde il ıo gennaio era stata spedita da Varchi il 27 dicembre: «Ho poi avuto per l'ultima vostra de' xxvij di decembre, alla quale ancora non ho risposto prima perché volevo scrivervi per via secura...» (CARO, Lettere familiari, n. [27-28 bis], par. 3).

i 2. Pietro Aretino, Lettere. Libro primo, a cura di P. Procaccioli, Roma, Salerno Editrice, I 997, n. 209 rr. I-I I. Del luogo ebbe contezza Girolamo Tiraboschi che nell'elenco delle donne amate da Molza mise anche questa ebrea (Biblioteca modenese, o notizie della vita e delle opere degli scrittori nati negli stati del Serenissimo Signor Duca di Modena, III, Modena, Presso la Società tipografica, 1783, p. 23 I; Storia della letteratura italiana, VII, 3, Modena, Presso la Società tipografica, I792, p. I I 36).

I 3. Molza, son. Qual empio ferro incenerir l'altezza (Serassi, III, n. II), 6-7: «del gran popol di Marte in quella pura»; canz. Signor, che 'n su'l fiorir de gli anni vostri (ibid., I, canz. VII), 2: «a la città del gran popol di Marte»; Petronio Barbati, Rime, Foligno, Campitelli, [17 I 2], LXXIX, I: «Superbo tempio il gran popol di Marte»; Giovanni Guidiccioni, Rime, a cura di E. Torchio, Bologna, Commissione per i testi di lingua, 2006, n. 60, Mentre che voi, cui vien dal ciel concesso, 3: «per honorar il buon popol di Marte». In Molza è però più frequente la formula "figliuol di Marte" per indicare Romolo, su impronta di Rvf 28, 79: «sai da l'imperio del figliuol de Marte»: Ninfa Tiberina, XXXIV, 7-8: «del figliuol di Marte / l'antiche mura» e i sonetti Alma gentil, che le gran membra sparte (Serassi, I, n. XXV), 8: «in sen ti rechi del figliuol di Marte»; Scipio, che lunge dal tuo patrio lido (ibid., n. LXIII), 2: «l'antiche mura del figliol di Marte»; Gli alti sepolcri et le mirabil spoglie (ibid., n. LXXV), 2: «del popol chiaro del figliuol di Marte»; Mentre il gran padre le reliquie sparte (ibid., II, n. XIII), 8: «alzar il popol del figliuol di Marte».

I4. VArchi, Lettere, p. 63. I nomi di Della Casa e Caro e anche di Claudo Tolomei ricorrono con toni confidenziali nella lettera a Molza della seconda metà di gennaio i 538 (ibid., p. 65 e cfr. supra la n. i i).

I 5. A. Gaspary, Storia della letteratura italiana, tradotta dal tedesco da Vittorio Rossi con aggiunte dell'autore, II, La letteratura italiana del Rinascimento, Parte seconda, 
Torino, E. Loescher, I 89 I (ed. or. Berlin I 888), pp. I 3 I-32. Rinviando espressamente a Gaspary, Francesco Flamini segnalò queste «rime che si dipartono, stante la novità del soggetto, dai modi comuni», in Il Cinquecento, Milano, Vallardi, I902, p. 55 I.

I6. Per questi due testimoni fondamentali delle rime molziane rinvio al mio F. Pignatti, Per l'edizione critica delle rime di Francesco Maria Molza. Il Casanatense 2667 e l'Ambrosiano Trotti 43I, in Lettura e edizione di testi italiani (secc. XIII-XX). Dieci progetti di dottorato di ricerca all' Università di Ginevra, a cura di M. Danzi, in «Quaderni ginevrini d'italianistica», 2 (2014), pp. I 23-205.

17. Giordani è forma etimologica dal latino Iordanim.

I 8. Con le seguenti rubriche: «Del Molsa à una Hebrea» (Poiche la vita ond'Israel fioria); «Del Medesimo alla medesima» (S'allhor che grave servitute oppresse).

19. Con le seguenti rubriche: «Secondo d'un'hebrea» (Poi che la vite ond'Israel fioriva); «Terzo alla medesima» (S'alhor che grave servitute oppresse).

20. BI Bologna, Archivio Isolani, capsula 95, F 69/i 66 (mutili nei versi iniziali a causa del fuoco); $\mathrm{CV}_{14}$ Città del Vaticano, Biblioteca apostolica, Vat. lat. $5172 ; \mathrm{F}_{\mathrm{I}}$ I fiori delle rime de' poeti illustri, nuovamente raccolti ed ordinati da Girolamo Ruscelli, Venezia, G.B. e M. Sessa, I 558 ; FN ${ }_{13}$ Firenze, Biblioteca nazionale, Magl. VII I I 92; $\mathrm{RD}_{3}$ Libro terzo de le rime di diversi nobilissimi et eccellentissimi autori nuovamente raccolte, Venezia, $\mathrm{Al}$ segno del pozzo, I 550 ; $\mathrm{RDR}_{\mathrm{I}}$ Rime di diversi eccellenti autori raccolte dai libri da noi altre volte impressi, Venezia, G. Giolito e fratelli, I 553; RSpB Rime spirituali di sette poeti illustri, Napoli, G. de Boy, I 569 ; RSpSa Rime spirituali di diversi eccellenti autori, Napoli, O. Salviani, I 574 ; $\mathrm{SI}_{5}$ Siena, Biblioteca comunale, I XI 49; $\mathrm{SI}_{6}$ Siena, Biblioteca comunale, $\mathrm{H} \mathrm{X}$ I; WR Wrocław, Biblioteka Uniwersitecka, Milich IV I 8 (Akz. I95 I, 289 [ı 8, 9350$]$ ).

2 I. Il sonetto è trascritto insieme con Alto Fattor del mondo, a cui non piace (di cui più avanti), e altri due di contenuto religioso eterogenei rispetto a quelli di cui stiamo trattando, da L. Felici, Frammenti di un dialogo. Ludovico Castelvetro e $i$ suoi rapporti con gli accademici modenesi, in Ludovico Castelvetro. Letterati e grammatici nella crisi religiosa del Cinquecento, Atti della XIII giornata Luigi Firpo Torino, 2I-22 settembre 2006, a cura di M. Firpo, G. Mongini, Firenze, Olschki, 2008, pp. 3 I 5-55, p. 34I n. 96.

22. Delle poesie volgari e latine di Francesco Maria Molra, II, n. CXLII.

23. Aggiungo alle sigle sciolte nella nota $2 \mathrm{I}$ RSpS Libro primo delle rime spirituali, Venezia, Al segno della speranza, I 550.

24. MV Modena, Biblioteca Estense universitaria, Raccolta Molza-Viti 27; $\mathrm{RN}_{1}$ Roma, Biblioteca nazionale centrale, Varia i 23 (728).

25. Ad esempio, Cariteo, canz. Candidi spirti, in ciel sempre fulgenti, vv. 31-33: «O notte, chiara più che 'l dì sereno / [...] / notte, in cui lo splendor vivo riluce» e Berardino Rota, canz. Donna, che prima del principio nostro, 36-37: «E ben fu quella notte a par del giorno, / anzi, d'ogni seren via più serena»; per altri riscontri cfr. Berardino Rota, Rime, a cura di L. Milite, Parma, Guanda, 2000, p. 5 i 9 n.).

26. Il sonetto è trascritto in Felici, Frammenti di un dialogo, cit., p. 34I n. 96. 